\title{
Vittorio Bodini, "Allargare il gioco». Scritti critici (1941-1970)
}

\author{
A cura di Antonio Lucio Giannone \\ Nardò: BesaMuci 2021.
}

Il volume, che raccoglie ventisei scritti di carattere critico e saggistico, preceduti da un'ampia introduzione e seguiti da un'accurata nota del curatore Antonio Lucio Giannone, costituisce un ulteriore tassello nella ricostruzione del percorso letterario di Vittorio Bodini. Tali interventi, pubblicati su periodici, riviste e quotidiani nell'arco di un trentennio, accompagnano in parallelo tutta la sua attività poetica e quella da ispanista, risultando particolarmente utili nell'ottica di una più profonda comprensione del suo itinerario creativo, se è vero che, parafrasando il Pavese de Il mestiere di vivere, nella lettura si ricercano "pensieri già da noi pensati", che offrendo delle conferme finiscano per far comprendere meglio le ragioni della propria ricerca.

Il periodo più fertile è quello tra il 1941 e il 1953, in cui Bodini comincia a collaborare con Oreste Macrì alla redazione della "terza pagina" del settimanale leccese Vedetta Mediterranea, prima di approdare, alla fondazione della propria rivista letteraria, L'esperienza poetica. Si tratta di una fase in cui, esaurita già da tempo la giovanile adesione al Futurismo e stabilito un contatto con i rappresentanti della "giovane letteratura" ermetica a Firenze, avvengono le fondamentali scoperte letterarie, che aiuteranno lo scrittore nella definizione dei propri nuclei poetici fondanti. Sono gli anni dell'apertura alla cultura europea, come testimoniano il Compianto di Joyce e le Opinioni su Poe e Kafka, che dimostrano "il tentativo del giovane scrittore di mettere in collegamento un'estrema provincia meridionale [...] con l'Europa" (p. 10), ma sono anche gli anni della "ricostruzione", durante i quali egli interviene attivamente nel dibattito sulla funzione della letteratura in un mondo ormai profondamente mutato, come quello del secondo dopoguerra.

In numerosi interventi critici, come in La cultura tradizionale e la "giovane letteratura", si delinea un'attenta riflessione sul ruolo della letteratura, alla ricerca di una strada diversa che potesse tenere in debita considerazione l'apertura verso le "impurità" della realtà, con cui era ormai necessario fare i conti, senza comunque "abdicare alle specificità del linguaggio letterario" (p. 14). La sua posizione si rivela piuttosto equilibrata, poco incline all'individuazione di schieramenti "politici" e all'attribuzione di "colpe", quanto piuttosto 
tesa alla ricerca di una conciliazione, in vista di una ricostruzione che fosse anche letteraria e permettesse alla letteratura italiana di riacquistare finalmente una "funzione europea" (p. 16). Una funzione che non può prescindere dallo scendere a patti con la realtà di ciò che è accaduto, con quella che in Invito alla retorica (con una nota sul gioco d'azzardo) viene definita un'aspirazione ad allargare il gioco: "Allargare il gioco in letteratura significa [...] includervi tutte le impurità, tutte le retoriche: e vedere poi se si è capaci di bruciarle" (p. 81), passando dunque anche per un rinnovamento del linguaggio, che per Bodini non dovrebbe mai ridursi alla semplice funzione mimetica e documentaria. D'altra parte, nella ricerca di una "terza via" tra ermetismo e neorealismo, in Mobili prospettive di una letteratura, sostiene: "Questo mondo noi non chiediamo sia fatto secondo questa o quella formula, ma che sia innanzi tutto, e poi che tenga conto di quanto è accaduto dentro di noi: ciò che basterebbe a garantirgli una viva impronta di umanità" (p. 103).

L'apertura al reale appare un leimotiv anche negli articoli critici dedicati in maniera più specifica alla poesia, tra cui spiccano Lettura montaliana e La poesía de Ungaretti en Allegria. Il primo costituisce una riflessione sulla presenza, nel passaggio dagli Ossi alle Occasioni, di un "tormentoso e vivace sentimento della polis" (p. 61). La poesía de Ungaretti en Allegria, invece, delinea i caratteri della raccolta ungarettiana, accompagnata dalla traduzione di alcune liriche dell'Allegria, destinata al pubblico spagnolo. In questa nota, Bodini mette significativamente l'accento sull'Ungaretti "uomo di pena" (p. 21 ), storicamente immerso nel suo tempo, seppur nella natura ontologica della sua poesia.

Negli anni Cinquanta, al rientro dalla Spagna, l'attenzione dell'autore si concentra attorno a nuovi poli d'interesse, tra cui spiccano la "scoperta del Sud" e la "cospirazione provinciale", come Bodini stesso la definì in un editoriale della sua Esperienza poetica. Infatti, "lo scrittore contrappone alla problematica nazionale che gli sembrava vuota e artificiosa, la concretezza, l'autenticità della provincia" (p. 23), un'operazione che tradurrà in forma poetica anche con le due "Lune". La scoperta del Sud campeggia anche nell'articolo dedicato a Quasimodo, le cui "parole - scrive - raggiunsero paesi e oggetti reali, che erano d'un territorio vergine nella geografia lirica italiana: il Mezzogiorno, anzi il Sud" (p. 155), diventando, nella prospettiva bodiniana, l'unico poeta civile dell'Italia della guerra e dell'occupazione.

Dopo gli anni dell'Esperienza poetica, l'attività critica si dirada, per lasciare posto a quella di ispanista e traduttore. A quest'ultimo periodo risale la Lettera a Carmelo Bene sul barocco, una risposta agli interrogativi sollevati da Bene sulle resistenze che ancora incontrava in Italia il barocco, come dimostrava l'utilizzo di una tale definizione per giustificare il rifiuto del suo film Don Giovanni. Bodini riconosce i pregiudizi che gravavano sul barocco come inevitabili segni di arretratezza culturale, dal momento che esso viene interpretato come "una condizione presente del nostro spirito" (p. 174), unica grande alternativa al mondo classico, scaturita dall'angoscia e da quell'horror vacui generato dalla 
perdita delle certezze e dei riferimenti tradizionali, di cui aveva già parlato, vent'anni prima, nella prosa Barocco del Sud.

Irene Pagliara 
